\title{
A COMPARATIVE STUDY OF RATE OF FALL OF TOTAL SERUM BILIRUBIN IN JAUNDICED BABIES FOLLOWING CONTINUOUS AND INTERMITTENT PHOTOTHERAPY
}

\author{
Bhatta B K*, Shrestha P S*
}

\begin{abstract}
Jaundice is observed during the first week of life in approximately $60 \%$ of term infants and $80 \%$ of preterm infants. ${ }^{1}$ Out of the $60 \%$ only $5 \%$ term and $20 \%$ preterm actually need treatment ${ }^{2}$ like phototherapy. The objective of the study was to calculate rate of fall of total serum bilirubin in jaundiced babies following continuous and intermittent phototherapy. A non-randomized, group matched, prospective, experimental study involving two comparable populations of 91 jaundiced babies receiving intermittent (45 babies - 6 hour on and 6 hour off) and continuous phototherapy ( 46 babies) were studied at the Tribhuvan University Teaching Hospital over a period of six months. The percent fall of total serum bilirubin for each 24-hour on first, second, third and fourth day of phototherapy was $-9.724 \%$ (SD 25.094), 4.717\% (SD 17.52), 7.468\% (SD 7.811) and 7.349\% (SD 4.482) respectively in continuous phototherapy. The corresponding figures for intermittent phototherapy were -13.623\% (SD 26.804), 1.131\% (SD 19.965), 7.672\% (SD 15.365) and 12.864\% (SD 8.29). The differences were statistically insignificant on the first, second and third day $(p=0.51, p=0.60$ and $p=0.95$ respectively). On the fourth day it was higher with the intermittent phototherapy $(p=0.04)$. The total duration of photo exposure was 64.435 hours (SD 22.251) in the continuous phototherapy group as compared to 35.581 hours (SD 12.855) in the intermittent phototherapy group $(p=0.000001)$. In view of the concern, which has been raised regarding the possible long-term side effects of phototherapy, intermittent phototherapy offers an attractive therapeutic alternative by reducing the duration of light exposure to about half without decreasing the efficacy.
\end{abstract}

Key Words: Neonatal jaundice, phototherapy.

\section{INTRODUCTION}

Jaundice is observed during the first week of life in approximately $60 \%$ of term infants and $80 \%$ of preterm infants. ${ }^{1}$ Out of the $60 \%$ only $5 \%$ term and $20 \%$ preterm actually need treatment, ${ }^{2}$ in the rest it disappears after a few days, often without any special treatment. Occasionally, hyperbilirubinaemia can reach levels toxic enough to cause permanent brain damage, which can then result in varying degree of neurological deficit and in extremely severe cases, even death.
The colour usually results from the accumulation in the skin of unconjugated, non-polar, lipid soluble bilirubin pigment (indirect-reacting) formed from haemoglobin by the action of haeme oxygenase, biliverdin reductase, and non-enzymatic reducing agents in the reticuloendothelial cells.

With the advent of phototherapy in $1958,{ }^{3}$ it has been accepted in neonatal units all over the world. Its noninvasive nature means that all complications attending an invasive procedure

\footnotetext{
* Manipal College of Medical Sciences, Pokhara, Nepal

** Tribhuvan University Teaching Hospital, Maharajgunj, Kathmandu, Nepal.

Address for correspondence : Dr. Bhubanesh Kumar Bhatta

Department of Paediatrics

Manipal College of Medical Sciences

P.O. Box: 155, Pokhara, Nepal

Email: bhubaneshbhatta@hotmail.com
} 
are eliminated. There are various regimens of giving phototherapy basically these can be divided into continuous and intermittent methods. The worry is that with easy availability of phototherapy, frequent exposure of infants to light will occur unnecessarily.

Phototherapy has potential side effects and concern has also been raised based on the lack of evidence of safety of phototherapy in the long run. The modification of phototherapy in which the duration of exposure of newborns to light can be reduced without compromising the efficacy would certainly be advancement over the conventional method. It is in this context that the present study was undertaken.

\section{MATERIAL AND METHODS}

\section{Study Design}

This is a non-randomized, group matched prospective, experimental study involving two comparable populations of babies receiving intermittent and continuous phototherapy.

\section{Setting}

This study was done in the Tribhuvan University Teaching Hospital, Neonatal unit from $11^{\text {th }}$ March 2000 to $10^{\text {th }}$ September 2000. All the newborns having jaundice, regardless of cause, who had total serum bilirubin levels which needed phototherapy were selected for the study.

\section{Group Matching}

To ensure comparability between the two groups they were selected in such a way that they are similar with regards to gestational age, birth weight, etc. which are known to influence the outcome and which if not adequately matched for comparability could distort or confound the results.

The newborn infants were divided into two groups closely matched and comparable with respect to gestational age, sex, birth weight, APGAR score at 1 minute and 5 minutes of birth, age at the start of the phototherapy and peak plasma bilirubin during the course of treatment.

The newborns were clinically assessed for jaundice in the morning rounds using Kramer's criteria. If it was thought to be higher for the age a TSB sample was drawn using the heel prick.

If the Total Serum Silirusin (TSB) came high enough in the range to start phototherapy using the chart in the neonatal unit, phototherapy was started after parental consent was taken.
Infants with the following conditions were excluded from the study:

1. Infants, who required exchange transfusion, blood / blood product transfusion, human albumin infusion during the period of the study.

2. Infants with conditions or anomalies (such as severe congenital CNS or heart malformations).

3. Infants who expired while still in the study.

The time at which phototherapy was started was taken as 0 hour (as there was some delay in getting the reports). TSB samples were drawn every 12 hourly following the start of phototherapy by doing a heel prick. $(0.2 \mathrm{ml}$ blood $)$

A heparinized capillary tube was used to collect blood. Measurements: W210xD256xH210mm.

The capillary tube was centrifuged using a high-speed haematocrit centrifuge manufactured by Kokusan Corporation, Tokyo, Japan. Kokusan centrifuge, Type H-1200 A.

TSB was measured by using BIL MICROMETER, Model A500, SN 3511469.

Phototherapy was provided by:ATOM PHOTOTHERAPY UNIT: 120 ST.

Four blue ATOM JET TSP 20W PHOTOTHERAPY FL 20 S-BW-NU tube lights were used.

Analysis was done using the Epi.info version 6 programme. The percent fall of plasma bilirubin per 24 hour and the mean fall in $\mathrm{mg} / \mathrm{dl} /$ day for every 24-hour of phototherapy was calculated for each infant by application of paired students ' $t$ ' test. The mean duration of phototherapy exposure was calculated and $\mathrm{p}$ value was calculated. A p value of $<$ or $=$ 0.05 was considered significant.

\section{RESULTS}

Table I shows the percent fall of TSB at 24-hour intervals. During the first 24 hour there was in fact an increase in the TSB in both the continuous and the intermittent phototherapy groups. ( $-9.274 \%$ and $13.623 \%$ respectively, $\mathrm{p}=0.51)$. During the $24-48$ hours period, the percent fall was $4.717 \%$ with the continuous phototherapy as compared to $1.131 \%$ with the intermittent phototherapy group but the difference was not significant statistically ( $\mathrm{p}=0.60$ ). During the $48-72$ hours period the percent fall was $7.468 \%$ with continuous phototherapy as compared to $7.672 \%$ with intermittent group but the difference was not significant statistically $(\mathrm{p}=0.95)$. During the $72-96$ hours the percent fall was $4.482 \%$ with continuous phototherapy as compared to $12.846 \%$ with intermittent group and the difference was significant statistically $(p=0.04)$. 
Table I : Percent fall of TSB at 24-hour interval

The total duration of phototherapy in the continuous phototherapy was 2964 hours as compared to 1530 hours in the intermittent phototherapy group.

The mean duration of phototherapy in the continuous group was 64.44 hours while it was 35.58 hours in the intermittent group. The difference was highly significant.

$(p<0.000001)$

Thus the same results were achieved with intermittent phototherapy while reducing the exposure time to almost half.

\section{DISCUSSION}

The first report on the use of phototherapy for treatment of neonates with jaundice was published more than 42 years ago. ${ }^{3}$ Since then phototherapy has been used extensively.

Present study also proves the effectiveness of phototherapy in controlling hyperbilirubinaemia in jaundiced babies. It was more effective after the first 24 hours of its use. There was an initial rise in the TSB in both the continuous and intermittent groups in the first 24 hour probably because of the initial rise of plasma bilirubin before the start of phototherapy which diluted the effect of phototherapy in the initial hours. Percent fall was significantly higher with intermittent phototherapy on the fourth day. This phenomenon may in part be because of movement of bilirubin load (photobilirubin, photoisomers) from skin and subcutaneous tissue and/or peripheral capillary wall into the blood stream pool, rather than delayed clearance of bilirubin and photoisomers from the blood stream to bile or urine. Brown $\mathrm{AK}$ et $\mathrm{al}^{4}$ demonstrated a similar pattern of greatest effect of phototherapy after the first day of phototherapy exposure. This study also demonstrates a declining efficacy after 72 hours in continuous phototherapy consistent with the hypothesis that unstable bilirubin isomers (photobilirubins) formed during the light treatment revert to natural bilirubin in the intestine following hepatic excretion and are reabsorbed via the enterohepatic circulation and contribute again to the bilirubin load to be cleared by the liver.

Over the years phototherapy has become the most important treatment for neonatal hyperbilirubinaemia. The question of subtle, long-term side effects, however, has not been adequately answered and it is theoretically important, therefore, that infants should be subjected to the least amount of irradiation without sacrificing the overall efficiency of the treatment. Various schedule of phototherapy have been investigated. Clinical studies comparing intermittent with continuous phototherapy have produced conflicting result. ${ }^{5,6,7}$

It was also strikingly apparent in this study that the mean duration of phototherapy was markedly reduced in the intermittent group 35.58 hour as compared to 64.44 hour in the continuous groups $(\mathrm{p}<0.000001)$. Similar findings have been observed in a study done by Govil YC and associates ${ }^{8}$ using continuous and intermittent (a 6 hour on and 6 hour off) phototherapy in two closely matched groups of 18 and 22 babies with hyperbilirubinaemia respectively, the percent fall of plasma bilirubin for each 24 hour was statistically not significant in the two groups. The total duration of phototherapy in the continuous group was 76 hour as compared to 40.36 hour in the intermittent phototherapy group $(\mathrm{p}<0.001)$. This slight difference may be because they used a locally improvised phototherapy unit using white lamps whereas in the present study special blue lamps were used. The mean duration of phototherapy in Osborn LM's ${ }^{9}$ study was 78.5 hours (range 57-84 hours) which is comparable to the continuous phototherapy group in the present study. 